\title{
12 Lead Placement Standard Extended Left
}

National Cancer Institute

\section{Source}

National Cancer Institute. 12 Lead Placement Standard Extended Left. NCI Thesaurus.

Code C71131.

An electrocardiogram (ECG) lead placement whereby the standard lead placement is

modified by having leads V7, V8 and V9. 